\title{
Content Accessibility Evaluation of Government Website using WCAG(Web Content Accessibility Guidelines)
}

\author{
Swikruti Dongaonkar \\ M.E.Scholar, Dept. Information \\ Technology Pimpri Chinchwad \\ College of Engineering \\ Pune, India. \\ Email: sudongaonkar@gmail.com
}

\author{
Ramkrishna Vadali \\ Professor, Dept. Information \\ Technology Pimpri Chinchwad \\ College of Engineering \\ Pune, India \\ Email: \\ ramkrishna.vadali1@gmail.com
}

\author{
Chandrakant Dhutadmal \\ Senior Technical Officer Centre \\ for Development of Advanced \\ Computing (C-DAC) \\ Pune, India \\ Email: cpdhutadmal@gmail.com
}

\begin{abstract}
A well designed and highly accessible websites are very important for organizations to communicate with different categories of the users in this IT world. It has also became mandatory for various organizations to design websites and offer all its services. Analysis of specific group of government websites across a spectrum shows that there was tremendous need for longitudinal study of website content accessibility. To accomplish this they put heaps of diligent work to represent information on sites which are client driven and can be open easily by the clients having distinctive capacities. This is possible with the help of continuous monitoring by the designers to design websites according to the website content accessibility standards i.e. WCAG(Web Content Accessibility Guidelines). The responsibility of designer or concerned organization is to evaluate the web content accessibility using web content evaluators. These evaluators will generate compliance reports according to which developers can modify their website content as per the need of every user. In this paper, the current scenario of various accessibility checkers and WCAG compliance for government website is analyzed.
\end{abstract}

Index Terms_WCAG; Compliance; Website Accessibility

\section{INTRODUCTION}

\section{Motivation}

A website is a combination of related website pages served through single area. Various types of websites are there however most generally utilized classes of the websites are government, instructive, business, social and employ- ment gateways. It has turned out to be required for an association to build up a website to outfit its administrations online through various systems. With increment in the quantity of websites and its prominence it is currently essential for an association to put lots of efforts to design the websites and its substance deliberately so that it can easily cater the requirements of various classes of clients with full accessibility.

Various web composition issues are there that should be kept into thought while outlining the website. One of the most critical issue among all is the web content accessibility[6]. Web content accessibility means removing barriers that prevents access to the people with different abilities so that they can recognize, understand, navigate, and interact with the content of the websites. Web content accessibility benefits others, including older people with changing abilities due to aging.

Some People with different abilities faces lot of problems to interact with the website and related web contents. Recent studies shows that contents of many websites are still not accessible to the people with different abilities[15][12]. A common observation is that the majority of the open-source systems were designed to be used by website developers who should have a solid background in the web accessibility and its guiding principle. Also, some web developers perform manual or automatic evaluations. In manual evaluation 
the developers manually examine web pages to find out problems. The developers might have potential bias while manually evaluating the accessibility quality of a web page[11].

\section{B.WCAG(Web Content Accessibility Guidelines) and Web}

\section{Content Evaluation}

WCAG (Web Content Accessibility Guidelines) is developed through the W3C (World Wide Web Consortium) process in collaboration with people and associations around the globe. The fundamental objective of WCAG is to demonstrate a solitary shared standard for web content accessibility that addresses the issues of governments, associations, and people, globally. WCAG 2.0 has 12 guidelines that are sorted out under 4 principles: perceivable, operable, understandable, robust. For every Guideline, there are testable achievement criteria, which are at three levels: A, AA, and AAA [16]. Web content accessibility evaluation is the procedure to verify the conformance of a website with the guidelines set by the standards organizations.

In this paper, the state of accessibility verification systems is evaluated. This paper focuses on the web content accessibility of government website and their compliance. The structure of the paper is as follows: In Section 2 literature is reviewed regarding web accessibility. Section 3 presents analysis and comparison of existing accessibility evaluation systems Section 4 presents proposed methodology. Section 5 concludes this paper and Section 6 includes the references.

\section{Literature Review}

There are many existing online accessibility evaluation tools to test the web Pages according to the WCAG Standards. These tools provide software support by analyzing HTML codes of a websites and also perform an analysis to identify usability problems depending on specific guidelines. More complicated tools may provide suggestions to improve the web sites quality. Some repair tools are also available which not only identifies the problems but also repair them automatically[9]. this literature review provides related research on Website Evaluators consisting of questionnaires. Specific automatic evaluation tools are introduced below.

AChecker: AChecker is an online accessibility evaluator, which assesses HTML pages as per the WCAG 1.0 and WCAG 2.0. AChecker is a semi-mechanized evaluator as it can't confirm all the guidelines of WCAG. Three types of errors are identified in AChecker namely known, likely and potential. Likely and Potential problems include the problems that AChecker require Human intervention to make a decision[13][10]. WAVE: WAVE is an evaluation tool developed by Web Aim. It gives a visual representation of the page being assessed with yellow notices which shows a mistakes. Warnings on the right side show accessibility issues. While those on the left side give an outline of the issues found. WAVE does not have a repair tool and does not show the implemented guidelines[7]. TAW: TAW is a free accessibility tool which is developed by the Spanish Foundation Centre for the Development of Information and Communication Technologies in Asturias (CTIC). TAW tests the accessibility according to WCAG 1.0 and 2.0. and its motivation is to analyze the accessibility of Web Design and Development for Access to each Individuals despite of their characteristic. The Accessibility problems produced by TAW are of three categories: problems, warnings, and "not reviewed." The "not reviewed" errors have no repair options. TAW does not show the implemented guidelines. HTML Tidy: HTML Tidy is a free evaluation tool which is fit for automatically settling basic HTML issues and distinguishing some potential accessibility problems[8]. HTML Tidy permits input HTML in three structures: as a URL, as direct HTML code written or pasted into text area, or as an uploaded file. This tool permits the user to choose several alternatives identified with the repairing procedure. Subsequent to uploading the HTML code and selecting the "Tidy" alternative, the repairs are done[3]. WAI(Web Accessibility Initiative): WAI was found by the World Wide Web Consortium (W3C) Web Accessibility[4], and enhances the availability of web for individuals having distinctive capacities[14]. 


\section{PROPOSED METHODOLOGY}

\section{A. Web Crawler}

A web crawler is an Internet Search Bot or a program which peruses the World Wide Web steadily. Fundamental motivation behind web crawler is web ordering or web spidering. Web search tools and some different sites utilize web creeping or web spidering programming to upgrade their web substance or records of others sites' web content. Web crawlers copy every one of the pages they visit for later handling by the search engine which indexes the downloaded pages so the clients can seek significantly.

\section{B. HTML Parser}

HTML Parser is only the software or Java library which is utilized to parse HTML in either a direct or settled design. It has two important purposes HTML traversal and HTML clean. It is principally utilized for transformation or extraction. HTML parser is a quick, strong and very well tested

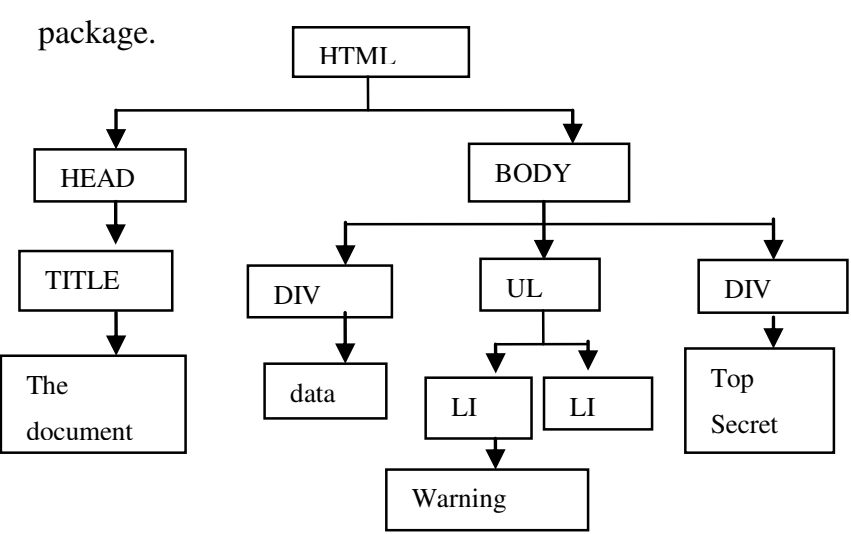

Fig. 1 HTML Parser

\section{DOM(Document Object Model) Parser}

The DOM is an official recommendation of the World Wide Web Consortium (W3C). DOM characterizes an interface that empowers programs to get access and redesign the structure, style, and contents of XML reports. The DOM is a typical interface for controlling document structures.

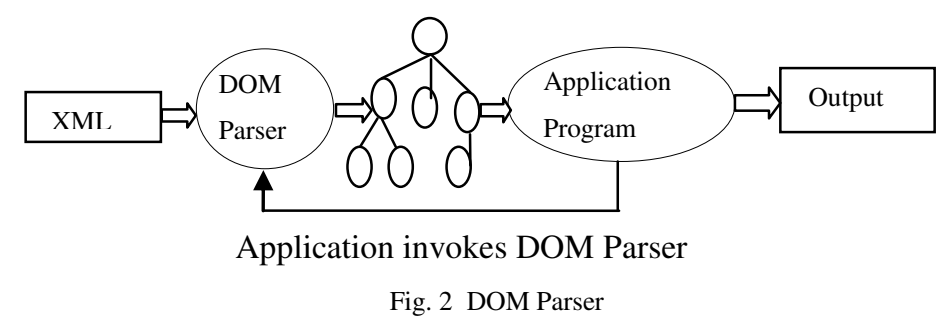

\section{Time Stamping}

A time stamp is the present time of an event that is recorded by the computer. Computer keeps up precise current time, adjusted to minute fractions of a second through components, for example, the Network Time Protocol ( NTP ).

\section{IV.ANALYSIS AND COMPARISON}

\section{A. WAVE And AChecker}

Name of the tool used: AChecker And WAVE

Technique Used: They collect the URLs of University Web Pages as an input and extract those URLs with the help of web scraping tool named 'import.io' from web Page. Websites homepages are evaluated with any of the tool such as AChecker and WAVE. After evaluation, websites are classified into three groups namely Low Accessible Websites called Tier-III, Medium Accessible called Tier-II and High Accessible Websites called Tier-I[1].

Advantage: Provide administrators, summarized results in terms of problems and also provides better solutions for errors[1].

\section{B. Accessibility Analyzer with JavaScript Algorithms}

Name of the tool used: Social4all Platform, JavaScript accessibility Analyzer

Technique Used: Social4all platform is framed, which permits an arrangement of an accessibility issues to be fathomed without adjusting the original page code. The primary expectation of this stage is to enhance the access of online information for individuals with various types of confinements. The proposed platform can investigate sites and distinguish numerous accessibility issues naturally [2].

Advantage: User can create its own adaptation profile. It also improves the accessibility of website[2].

\section{IWAET(Interactive Evaluation Tool)}

Name of the tool used: IWAET(is an extension of AChecker) 
Technique Used: IWAET consist of five modules:

1) Parse module: Accessibility results in HTML format are taken. Parsing algorithm accesses the HTML files and gets the content of the HTML tags and saves them into an array of results.

2) Unclear module: It replaces no clear description accessibility errors with simple, understandable, and clear words.

3) Redundant module: Merges redundant errors so only one error is produced. It is based on IWAET database.

4) Repair module: It is used for fixing of errors.

5) Results module: It stores the accessibility problems encountered, each described by its id, description, and fixed code[3].

Advantage: Simple and attractive Graphical User Interface. IWAET display results in tabular format containing the error number, the error description and the violating code[3].

\section{TAW And Webometrics}

Name of the tool used: TAW and Webometrics

Technique Used: The specialized assessment is done utilizing TAW and non-specialized assessment through direct perception utilizing webometrics achievement criteria. Technique and assessment stages is isolated into 5 phases [4]:

1) The Literature Study and Information Collection: It incorporates literature study about the webometric positioning and web assessment, comprise of web assessment tool and web accessibility.

2) Examination of Web based-on Criteria Webometrics: Literature study recognize the issue criteria which are examined, in view of the webometrics ranking technique.

3) Mapping to WCAG 2.0 Criteria of Webometrics: Webbased assessment criteria were mapped into the assessment criteria of WCAG 2.0.

4) Classification Criteria of the Webometrics: Grouping of criteria is done in two technical and non-technical. Technical criteria contains web specialized appraisal assessment of webometrics criteria with WCAG 2.0, while the assessment of the non-technical criteria is impossible with WCAG 2.0 however done through the social evaluation.

5) Assessment Criteria for Technical and Non-Technical: The technical assessment of the criteria was done utilizing a web assessment test device or tool and Non-technical criteria were assessed by direct perceptions of the site.

Advantage: Technical and non-technical evaluation to improve the university website[4].

\section{E. Accessibility Analyzer}

Name of the tool used: Own Accessibility Analyzer

Technique Used: Techniques are based on the qualitative method of data collection consisting of four steps[5]:

1) Choosing the profile of the clients participating in the research: Adults who were enlisted up to the fourth grade, as per the UNESCO arrangement are picked.

2) Performance of an ethnographic study: This progression is utilized to get some answers concerning the behavior of the chose individuals and analyze their association with the computers.

3) Execution of a case study which comprised in analysing functionally illiterate persons: This step comprise of two stages. To begin with is Evaluation with clients who were not practically uneducated and the second one is Evaluation with practically unskilled clients.

4) Justification of the rules: The outcome was produced as a rundown of those qualities that add to adjustment of usability assessment strategies with functionally illiterate users [5]. Advantage: Increase the usability for functionally illiterate users[5].

\section{V.CONCLUSION}

With a specific end goal to better present the website content accessibility aspect, web specialists must have to consider web content accessibility guidelines(WCAG) standards. With the result analysis of existing website content accessibility, it is very clear that majority share of the website classifications are not too much worried about giving content accessibility highlight in their website and optional approach for web content for individuals with various capacities. In 
this research work, compliance validation of government websites is proposed using web content accessibility guidelines(WCAG). The main aim of web content accessibility evaluator is to provide uniform access to each user for using any websites. Application proposed in the context of this research facilitates the evaluation process and provides information to website owners to realize the deficiencies of their websites. It also gives a comparison with its rival websites to determine a roadmap to take necessary measures.

\section{REFERENCES}

[1] Abid Ismail, K. S. Kuppusamy, "Accessibility of Indian universities' home pages: An exploratory study", Journal of King Saud University Computer and Information Sciences (2016) science direct 2016.

[2] Rubén Gonzalez Crespo, Jordán Pascual Espada, Daniel Burgos "Social4all: Definition of specific adaptations in Web applications to improve accessibility", Computer Standards and Interfaces 48 (2016) 1-9 Elsevier 2016

[3] Islam Elkabani Lama Hamandi, Rached Zantout, Simar Mansi, "Toward Better Web Accessibility", Information \& Communication Technology and Accessibility (ICTA), 2015 5th International Conference, 2015.

[4] Paramaresthi Windriyani, Ridi Ferdiana, Warsun Najib, "Accessibility Evaluation Using WCAG 2.0 Guidelines Webometrics Based Assessment Criteria (Case Study: Sebelas Maret University)", ICT for Smart Society(ICISS) 2014 International Conference, 2014.

[5] Eliane Pinheiro Capra, Simone Bacellar Leal Ferreira, Denis Silva da Silveira,Ariane Oliveira Ferreira, "Evaluation of Web Accessibility: an Approach Related to Functional Illiteracy", Procedia Computer Science 14 ( 2012 ) 36 - 46 Elsevier, 2012.
[6] Jatinder Manhas, "Design and Development of Automated tool to Study Sitemap as Design Issue in Websites", 2015 2nd International Conference on Computing for Sustainable Global Development (INDIACom), 2015.

[7] WAVE-web accessibility evaluation tool, www.wave.webaim.org, Retrieved 1-10- 2014.

[8] Tidy, http://tidy.sourceforge.net/docs/quickref.html, Retrieved 12122014.

[9] N. Fernandes, D. Costa, C. Duarte, L. Carriço, "Evaluating the accessibility of web applications", Proceedings of the 13th international conference on Computers Helping People with Special Needs, Vol. part 1, p. 369-403, 2012

[10] Gay G, Qi Li C, "AChecker: Open, Interactive, Customizable, Web Accessibility Checking", W4A '10 In Proceedings of the 10th International Cross Disciplinary Conference on Web Accessibility, (W4A), New York, NY, USA, Article 23, 2010.

[11] Lopes R, Van Isacker K, Carriço L, "Redefining assumptions: Accessibility and its stakeholders", In The 12th International Conference on Computers Helping People with Special Needs (ICCHP). Vol. 6179, p. 561-568, 2010.

[12] Kern, WEB 2.0 - End of accessibility analysis of most common problems with WEB 2.0, International Journal of Public Information Systems, Vol. 4, 2008.

[13] Fukuda K, Saito S, Takagi H, Asakawa C, "Proposing New Metrics to Evaluate Web Usability for the Blind", Processing CHI '05 Extended Abstracts on Human Factors in Computing System, p. 1387-1390, 2005.

[14] https://en.wikipedia.org/wiki/Web_Accessibility_Initiative.

[15] Burgstahler S, Jirikowic T, Kolko B, Eliot M, "Software Accessibility, Usability Testing and Individuals with Disabilities", Information Technology and disabilities Journal, Vol. 10(2), 2004.

[16] https://www.w3.org/WAI/intro/wcag.php. 